\title{
A Car-Following Model Based on Safety Margin considering ADAS and Driving Experience
}

\author{
Yugang Wang $\mathbb{C}^{1,2}$ and Nengchao Lyu $\mathbb{D}^{1,2}$ \\ ${ }^{1}$ Intelligent Transportation Systems Research Center, Wuhan University of Technology, Wuhan 430063, China \\ ${ }^{2}$ National Engineering Research Center for Water Transport Safety, Wuhan 430063, China \\ Correspondence should be addressed to Nengchao Lyu; lnc@whut.edu.cn
}

Received 16 October 2020; Revised 29 January 2021; Accepted 8 February 2021; Published 19 February 2021

Academic Editor: Hui Yao

Copyright (c) 2021 Yugang Wang and Nengchao Lyu. This is an open access article distributed under the Creative Commons Attribution License, which permits unrestricted use, distribution, and reproduction in any medium, provided the original work is properly cited.

\begin{abstract}
Existing studies had shown that advanced driver assistance systems (ADAS) and driver individual characteristics can significantly affect driving behavior. Therefore, it is necessary to consider these factors when building the car-following model. In this study, we established a car-following model based on risk homeostasis theory, which uses safety margin (SM) as the risk level quantization parameter. Firstly, three-way Analysis of Variance (ANOVA) was used to analyze the influencing factors of car-following behavior. The results showed that ADAS and driving experience have a significant effect on the drivers' car-following behavior. Then, according to these two significant factors, the car-following model was established. The statistical method was used to calibrate the parameter reaction response $\tau$. Other four parameters $\left(\mathrm{SM}_{\mathrm{DL}}, \mathrm{SM}_{\mathrm{DH}}, \alpha_{1}\right.$, and $\left.\alpha_{2}\right)$ were calibrated using a classical genetic algorithm, and the effects of ADAS and driving experience in these four parameters were analyzed using $T$-test. Finally, the proposed model was compared with the GHR model, and the result showed that the proposed model has a smaller Root Mean Square Error (RMSE) than the GHR model. The proposed model is a method of simulating different driving behaviors that are affected by ADAS and individual characteristics. Considering more driver individual characteristics, such as driving style, is the future research goal.
\end{abstract}

\section{Introduction}

With the increasing number of vehicles, societies have been faced with significant challenges to congestion and traffic crashes. A recent report from World Health Organization [1] indicated that, annually, 1.24 million people die due to traffic crashes. Human factors are the most important cause of traffic accidents, and driver behavior plays a leading role in safe driving. Car-following behavior is one of the most common and important behaviors in the driving process, and it is closely related to traffic safety. Researchers have been studying the car-following process for more than half a century and have established a number of car-following models that focus on traffic flow theory, control algorithms, or driver psychology and physiology. These car-following models are important to ADAS, especially the ACC system $[2,3]$, and are considered to be key assessment tools for transportation systems $[4,5]$. The existing car-following model mainly includes the CA model (collision avoidance model), which is based on maintaining a safe braking distance, and the GM model (stimulus-response model), which is based on a stimulus-response mechanism. The CA model calculates the specific theoretical safety following distance combined with vehicle braking performance and driver response time by using Newton's law of motion [6]. The GM model uses the relative speed between the leading and host vehicle as the stimulus standard, uses the host vehicle's acceleration and deceleration as the reaction situation, and introduces the sensitivity as the adjustment coefficient to study the relationship between the parameters in the carfollowing process [7]. These evaluation models effectively simulate the car-following behavior and determine how the car-following occurs in the actual scenario, but the reasons for the vehicles to follow each other in some way are not 
discussed in depth. Hamdar et al. explained car-following behaviors based on the prospect theory and proposed a carfollowing model by evaluating the gains and losses while driving [8]. Wilde's risk homeostasis theory (RHT), another risk-taking theory, is also helpful in explaining car-following behaviors [9]. A car-following model based on risk perception, which is RHT and stimulus-response mechanism, helps understand car-following mechanisms and facilitate the simulation of car-following behaviors that differ depending on varied individual characteristics, which have different levels of acceptable risk [10].

To improve drivers' attention and perceptual ability, various Advanced Driver Assistance Systems (ADAS) were developed to provide drivers with correct preaccident human errors. ADAS are designed to complement driver capabilities for perception, cognition, action selection, and action implementation in a dynamic environment $[11,12]$. For the influence of ADAS on driving behavior, many scholars have carried out research from different aspects. Blaschke et al. [13] tested the effectiveness of ADAS through Field Operational Tests (FOT) and found that the driver can more accurately estimate the distance to the leading vehicle after using ADAS and can maintain a safer time headway (THW) while driving. Saito et al. [11] conducted a simulated driving experiment to test the performance of ADAS through drivers awakening status and lane keeping performance. The results show that ADAS helps reduce traffic accidents, which is related to fatigue driving. Birrell et al. [14] tested ADAS through FOT. In their tests, after using ADAS, the driver's fuel efficiency increased by $4.1 \%$, and the average THW increased to 2.3 seconds. Adell et al. [15] studied the effects of ADAS on the safety distance and safe speed of drivers through FOT. The results showed that both positive and negative effects existed. Lyu et al. [16] studied the effect of driving experience on the effectiveness of ADAS through FOT. The results showed that ADAS had a positive impact on skilled drivers and unskilled drivers, especially for unskilled drivers, in the following vehicle scenario. But in the braking scenario, ADAS had a positive impact on unskilled drivers but a negative impact on skilled drivers. Lyu et al. [17] evaluated the effectiveness of ADAS in improving driver's risk perception in near-crash events using a novel metric from risk homeostasis theory. The results show that ADAS has a positive impact on the low-risk group and moderate-risk group for all drivers, but a negative impact on the high-risk group for skilled drivers. These studies show that ADAS can significantly affect the driver's acceptable risk level, so it is necessary to consider the impact of ADAS when establishing a car-following model.

In addition, driver characteristics can significantly affect the effectiveness of ADAS, such as gender [18] and driving experience [19]. Existing studies had shown that driving experience has an impact on driving performance. Rich driving experience can make the excellent driving performance, which is related to the driver's learning process and experience [20]. Compared with skilled drivers, unskilled drivers take up more attention resources and cause higher workload [21], and the accident risk is 2-4 times higher [22]. In addition, gender is one of the most commonly measured variables in driving behavior researches, and it has been identified as a key demographic variable that affects driving performance [23]. Therefore, the driver characteristics should also be considered when establishing a car-following model.

This article analyzes which driver characteristics can significantly affect the driver's car-following behavior firstly. Then, we establish the car-following model based on significant influencing factors.

\section{Database and Preparation}

2.1. Field Operational Tests Design. Driving simulator [11] and Field Operational Tests [14] approaches were employed to study the influences of ADAS to driver behavior. With the development of intelligent vehicles and the application of vehicle sensors, it is becoming a trend to evaluate the effect of ADAS with Field Operational Tests. Hence, an instrumented vehicle, which is equipped with ADAS, was developed to carry out Field Operational Tests on a variety of roads in Wuhan, China.

44 participants (24 males, 20 females) were recruited in these Field Operational Tests. Their age ranged from 22 to 55 years old (mean $=32.2 ; \mathrm{SD}=8.2$ ). On average, they had 6.9 years of driving experience, ranging from 2 to 18 years. It should be noted that, in China, many people obtain their driver's license but do not obtain actual driving experience. In order to avoid interfering with the credibility of the experiment, this research used a driver's total driving mileage rather than his/her driving license age as an indicator of proficiency. In terms of driving mileage, participants drove an average of 110,000 kilometers, ranging from 400 to 400,000 kilometers. In this study, drivers with a total driving range of more than 40,000 kilometers were defined as skilled drivers, while those with less than 40,000 kilometers were defined as unskilled drivers. There were 22 skilled drivers and 22 unskilled drivers in the recruited participants.

An instrumented vehicle has been developed to collect complete coverage of data on drivers' behavior, vehicle kinematics, and vehicle surroundings. The main equipment of the instrumented vehicle is shown in Figure 1. Mobileye was used to obtain headway and lane position, as well as to alert forward collision and lane departure. One video camera was used to collect vehicle surroundings video information. The INS system was used to obtain the vehicle's three-axis acceleration, latitude, and longitude. Lidar was used to obtain the forward target information (distance, velocity, relative velocity, etc.). The CAN bus was used to obtain vehicle data such as vehicle speed, accelerator pedal information, brake pedal information, and steering wheel angle. All data was synchronized with the master time that was transmitted by the monitoring software every $0.1 \mathrm{~s}$.

2.2. Extraction of the Car-following Data. Based on LiDAR and CAN bus data, the car-following segments were extracted. Table 1 summarized the rules used to extract the car-following segments from existing studies. 


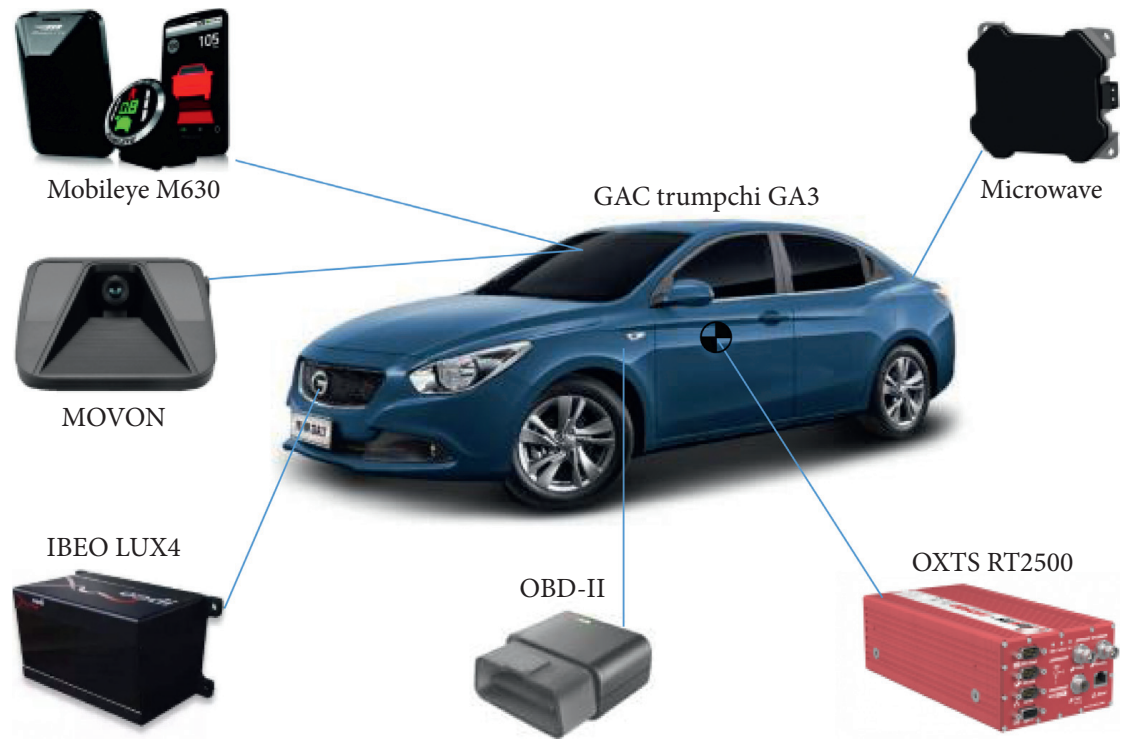

FIGURE 1: Equipment of instrumented vehicle.

TABLE 1: Summary of car-following event extraction criteria in the existing literature.

\begin{tabular}{|c|c|c|c|c|c|}
\hline & $\begin{array}{c}\text { Absolute lateral } \\
\text { distance }(\mathrm{m})\end{array}$ & $\begin{array}{l}\text { Host vehicle } \\
\text { speed }(\mathrm{km} / \mathrm{h})\end{array}$ & Longitudinal distance $(\mathrm{m})$ & Relative velocity $\left(\mathrm{m} / \mathrm{s}^{2}\right)$ & Segment duration (s) \\
\hline Leblanc et al. [24] & & $>40$ & & $<2.0$ & $>15$ \\
\hline Chong et al. [25] & $<1.9$ & $>20$ & $<120$ & & $>30$ \\
\hline Ervin et al. [26] & & $>40$ & & $<2.0$ & \\
\hline Wang et al. [27] & $<2.5$ & $>18$ & $>7$ and $<120$ & $<2.5$ & $>15$ \\
\hline
\end{tabular}

The key to the extraction of car-following segments is the setting of the threshold values. Due to the differences in traffic environment and driving behaviors between domestic and foreign, the threshold value in the literature could only be used as a reference, and it needs to be revised according to the data of these FOTs. According to the distribution range of each parameter in the FOTs data and the threshold values in the existing literature, the threshold values of the various car-following extraction rules in this study are determined as follows:

(1) Absolute lateral distance is $<2.5 \mathrm{~m}$, which ensures that the target vehicle is in the same lane as the experimental vehicle.

(2) The longitudinal distance is $<120 \mathrm{~m}$, and the host vehicle speed is $>20 \mathrm{~km} / \mathrm{h}$. These two rules can eliminate traffic flow conditions of congestion and free flow, and the congestion is excluded when the host vehicle speed $>20 \mathrm{~km} / \mathrm{h}$. The distance between two vehicles in free flow is too large, in which case the leading vehicle cannot restrict the driving behavior of the host vehicle, so a longitudinal distance $<120 \mathrm{~m}$ is required.

(3) The absolute value of longitudinal relative velocity is $<2.5 \mathrm{~m} / \mathrm{s}^{2}$, and the absolute value of relative velocity is small enough to ensure that the vehicle is in a stable car-following state.
(4) The segment duration should be more than 30 seconds to ensure the stability of the car-following status.

According to the above four extracting thresholds, 354 car-following segments were extracted from FOTs.

2.3. Risk Perception Quantification Index. We need a risk level quantitative parameter to describe the driver's risk perception changes in the car-following process. Commonly used risk level quantization parameters are Time Headway (THW) and Time to Collision (TTC). But THW and TTC have limitations as traditional indicators of risk quantification. THW retains only the vehicle speed information and does not consider the impact on relative speed, while TTC retains only the relative speed information and does not take into account the impact of the vehicle speed. Additionally, the expected means of THW and TTC are quite different between drivers with different characteristics [28] Therefore, there is a need for an indicator that is consistent between drivers with different characteristics to evaluate the effectiveness of ADAS. This indicator should take into account the impact of speed and relative speed. The safety margin proposed by the risk homeostasis theory (RHT) [29] is a suitable indicator. Nilsson [30] suggested that "everything in the driver's visual field that could be an obstacle or a threat is surrounded by zones, that is, 
safety margins." In the driver's control system, the safety margin $(\mathrm{SM})$ is defined as the threshold that protects the driver from danger [17]. The SM is independent of the actual driving situation. In accordance with that definition, the SM should have the following features: (a) it should be a monotonic function of the objective risk level; (b) drivers should have a fixed acceptable SM to guide their behaviors; and (c) an acceptable SM varies depending on the driver's characteristics; however, a certain type of driver (such as a highly skilled driver) should have a similar acceptable SM.

According to the barking process of the car-following task, the risk level can be defined as [31]

$$
\xi(\tau, t)=\frac{V_{1}(t) \cdot \tau+\left(\left[V_{1}(t)\right]^{2} / 2 a_{1}(t)\right)-\left(\left[V_{2}(t)\right]^{2} / 2 a_{2}(t)\right)}{D_{1}(t)} .
$$

In the above formula, $V_{1}(t)$ is the host vehicle's speed. $V_{2}(t)$ is the lead vehicle's speed. $a_{1}(t)$ is the car-following's acceleration; $a_{2}(t)$ is the lead vehicle's acceleration; $D_{1}(t)$ is the relative spacing between the two vehicles. $\tau$ is the reaction time, which consisted of the driver's reaction time $\tau_{1}$ and the braking system's reaction time $\tau_{2}$. This formula shows that when one vehicle was following another, the lead vehicle emergency brake could be applied until its speed dropped to zero. The driver of the car-following could observe the brake light signal of the lead vehicle and respond quickly. The distance the car-following passes is expressed as $V_{1}(t) \cdot \tau+\left(\left[V_{1}(t)\right]^{2} / 2 a_{1}(t)\right)$, and the distance the lead vehicle passes is expressed as $\left[V_{2}(t)\right]^{2} / 2 a_{2}(t)$. In this case, the quantitative objective driving risk level can be expressed as the difference between the distance and the relative spacing at time $t$. In this study, the formula was changed based on elements of driver and vehicle system factors:

$$
\xi(\tau, t)=\xi\left(\tau_{2}, t\right)+\frac{V_{1}(t) \cdot \tau_{1}}{D_{1}(t)} .
$$

Basing driving simulator on that definition, traffic safety can be ensured when $\xi(\tau, t) \leq 1$. It is important to note that, in a real-world scenario, it is possible that $\xi(\tau, t)>1$; however, no accident will occur in some circumstances. This is because the absolute value of the acceleration of the car-following is greater than that of the lead vehicle. However, if the lead vehicle brakes by the maximum deceleration at this time, a rear-end crash will still occur even if the car-following brakes at the maximum deceleration.

Therefore, under ideal conditions,

$$
\frac{V_{1}(t) \cdot \tau_{1}}{D_{1}(t)} \leq 1-\xi\left(\tau_{2}, t\right) .
$$

From here, the SM can be defined as

$$
\operatorname{SM}(t)=1-\xi\left(\tau_{2}, t\right) .
$$

According to previous research, the limit value of the acceleration of the vehicle is $0.3 \mathrm{G}-0.75 \mathrm{G}$ [32]. Combining the FOTs on the actual road, deceleration in the calculation of risk was set as

$$
a_{1}(t)=a_{2}(t)=7.0 \mathrm{~m} / \mathrm{s}^{2} .
$$

Braking system response time is usually between 0.10 and $0.30 \mathrm{~s}$. During emergency situations, a driver may quickly and strongly apply the brakes. Thus, $\tau_{2}$ was set as $0.15 \mathrm{~s}$.

Therefore, the SM can be calculated by the following formula:

$$
\operatorname{SM}(t)=1-\xi(0.15, t)=1-\frac{0.15 * V_{1}(t)+\left(\left[V_{1}(t)\right]^{2} / 14\right)-\left(\left[V_{2}(t)\right]^{2} / 14\right)}{D_{1}(t)} .
$$

According to the definition, the smaller the SM is, the higher the objective risk level is, which means that the risk of rear-end is higher. In the next section, each car-following segment will calculate the mean value of SM. Based on the ANOVA of the mean value of SM, the significant influencing factors to driver's car-following behavior can be obtained.

2.4. Factors Affecting Car-Following Behavior. Before the analysis of variance, judging by box figure, there is no abnormal data value, and after Shapiro-Wilk test, all groups of $\mathrm{SM}_{\text {mean }}$ are of normal distribution $(P>0.05)$. Furthermore, $P$ value of Levene homogeneity test of variance is 0.130 , which is greater than 0.05 , and it is believed that the dependent variables in each classification have equal variance. According to the above test, the data obey normal distribution, have no abnormal value, and have equal variance, so the three-way ANOVA can be analyzed.

The results of three-way ANOVA are shown in Table 2.

The results of three-way ANOVA showed that ADAS, gender, and driving experience did not have the interaction between three factors on the $\mathrm{SM}_{\text {mean }}, F(2,129)=0.454$, $P=0.636$. In the interaction between two factors, the influence of $\mathrm{ADAS}^{*}$ gender on the dependent variable was not statistically significant, $F(1,129)=0.824, P=0.193$; the influence of ADAS*driving experience on the dependent variable was statistically significant, $F(1,129)=0.001$, $P=11.841$; the influence of gender* driving experience on the dependent variable was not statistically significant, $F(1$, $129)=0.505, P=0.688$. In the main effect, ADAS has no main effect on the dependent variable, $F(1,129)=0.017$, $P=0.896$; the gender has no main effect on the dependent 
TABLE 2: Three-way ANOVA results for $\mathrm{SM}_{\text {mean }}$.

\begin{tabular}{|c|c|c|c|c|c|}
\hline Source & Type III sum of squares & $\mathrm{df}$ & Mean square & $F$ & Sig \\
\hline ADAS & 0.000 & 1 & 0.000 & 0.017 & 0.896 \\
\hline Gender & 0.075 & 1 & 0.037 & 2.538 & 0.085 \\
\hline Driving experience & 0.016 & 1 & 0.016 & 4.159 & $0.043^{*}$ \\
\hline ADAS* gender & 0.002 & 1 & 0.001 & 0.193 & 0.824 \\
\hline ADAS* driving experience & 0.046 & 1 & 0.046 & 11.841 & $0.001^{* *}$ \\
\hline Gender* driving experience & 0.005 & 1 & 0.003 & 0.688 & 0.505 \\
\hline ADAS* $^{*}$ gender ${ }^{*}$ driving experience & 0.004 & 2 & 0.002 & 0.454 & 0.636 \\
\hline
\end{tabular}

${ }^{* * *}$ Sig. $<0.001,{ }^{* *}$ sig. $<0.01$, and ${ }^{*}$ sig. $<0.05$.

variable, $F(1,129)=2.538, P=0.085$; the driving experience has a main effect on the dependent variable, $F$ (1, 129) $=4.159, P=0.043$.

The results of ANOVA showed that only driving experience can significantly affect the driver's car-following behavior, but in the interaction between two factors, the influence of $\mathrm{ADAS}^{*}$ driving experience on the dependent variable was statistically significant. Therefore, the effect of ADAS on the car-following behavior cannot be ignored. The subsequent model will take into account the factors of ADAS and driving experience.

\section{Car-Following Model Based on Risk Perception}

3.1. Model Assumptions. When the driver follows another vehicle, he will adjust the relative spacing to ensure an acceptable level of risk. That is, the driver of the following vehicle responds to the difference between the perceived safety margin (perceived risk level) and the desirable safety margin (DSM), as the driver of the following vehicle decides to accelerate (perceived safety margin is greater than DSM), decelerate (perceived safety margin is less than DSM), or maintain a constant speed (perceived safety margin is within DSM) [10]. Since the proposed model is based on the difference between the driver's DSM and the perceived safety margin, it is called the DSM model.

The DSM model for car-following is described as follows:

$$
a_{n}(t+\tau)=f\left(\mathrm{SM}(t)-\mathrm{SM}_{D}\right)= \begin{cases}\alpha_{1}\left(\mathrm{SM}(t)-\mathrm{SM}_{\mathrm{DH}}\right), & \mathrm{SM}(t)>\mathrm{SM}_{\mathrm{DH}} \\ \alpha_{2}\left(\mathrm{SM}(t)-\mathrm{SM}_{\mathrm{DL}}\right), & \mathrm{SM}(t)<\mathrm{SM}_{\mathrm{DL}} \\ 0, & \text { else, }\end{cases}
$$

where $a_{n}(t)$ is the acceleration of a following car at time $t$. $\mathrm{SM}_{\mathrm{DH}}$ is the upper limit of the DSM, and $\mathrm{SM}_{\mathrm{DL}}$ denotes the lower limit of the DSM. The goal of our modeling is to describe natural driving, and DSM may change due to psychological factors. However, in the simulation used to analyze the main factors affecting the automatic tracking process, the random factors can be ignored, so in the specific traffic environment, for the same type of driver, $\mathrm{SM}_{\mathrm{DH}}$ and $\mathrm{SM}_{\mathrm{DL}}$ can be constant values. $\alpha_{1}$ and $\alpha_{2}$ are the sensitivity factors for acceleration and deceleration, where $\alpha_{1}>0$, $\alpha_{2}>0$.

According to previous research, the limit value of the acceleration of the vehicle is $0.3 \mathrm{G}-0.75 \mathrm{G}$ [30], so the maximum deceleration is set as $7.0 \mathrm{~m} / \mathrm{s}^{2}$. If $a_{n}(t)<-7.0 \mathrm{~m} / \mathrm{s}^{2}$, then $a_{n}(t)=-7.0 \mathrm{~m} / \mathrm{s}^{2}$. Maximum favorite acceleration is set as $3.0 \mathrm{~m} / \mathrm{s}^{2}$. If $a_{n}(t)>3.0 \mathrm{~m} / \mathrm{s}^{2}$, then $a_{n}(t)=3.0 \mathrm{~m} / \mathrm{s}^{2}$.

Because of the simulated time interval, the result of calculated speed may be negative when the speed is close to zero in numerically updated process. Thus, the minimum speed of the following car is set as $V(t)=0.0 \mathrm{~m} / \mathrm{s}$, if $V_{n}(t)$ $<0.0 \mathrm{~m} / \mathrm{s}, V_{n}(t)=0.0 \mathrm{~m} / \mathrm{s}$.
3.2. Model Calibration. According to the car-following model, a total of five parameters need to be calibrated: response time $\tau$, DSM lower limit $\mathrm{SM}_{\mathrm{DL}}$, DSM upper limit $\mathrm{SM}_{\mathrm{DH}}$, acceleration sensitivity coefficient $\alpha_{1}$, and deceleration sensitivity coefficient $\alpha_{2}$.

The response time is the reaction time of the following vehicle. According to the existing research, the reaction time in the car-following process can be determined by comparing the relative speed curve and the acceleration curve of following vehicle [33]. According to the theory of the stimulus-response model [34], the acceleration of the following vehicle is determined by the relative speed of the two vehicles; that is, the relative speed change (stimulus) will cause a corresponding change (reaction) of the following vehicle's acceleration after a certain time delay, and this delay is the car-following reaction time.

From the extracted 354 car-following events, the reaction time of each pair of stimulus-response points was extracted, and the mean value was calculated according to whether or not to use ADAS and driving experience, respectively. When ADAS is off, the average response time of 
unskilled drivers is $1.5 \mathrm{~s}$, and the average response time of skilled drivers is $1.3 \mathrm{~s}$. This is because, without the support of ADAS, unskilled drivers need to take more attention resources and cause higher workload when handling driving tasks, so the response time is slower than that of skilled drivers. After exposure to ADAS, the average response time of unskilled drivers is $1.0 \mathrm{~s}$, and the average response time of skilled drivers is $0.9 \mathrm{~s}$. After exposure to ADAS, the response time of skilled drivers and unskilled drivers will be shortened, and the change of unskilled drivers is more obvious. This phenomenon indicates that ADAS has more influence on skilled drivers than unskilled drivers, consistent with the existing research [35].

The remaining four parameters, DSM lower limit $\mathrm{SM}_{\mathrm{DL}}$, DSM upper limit $\mathrm{SM}_{\mathrm{DH}}$, acceleration sensitivity coefficient $\alpha_{1}$, and deceleration sensitivity coefficient $\alpha_{2}$, cannot be directly derived using statistical methods; therefore, we denote them as a vector, $\mathbf{V}=\left(\mathrm{SM}_{\mathrm{DL}}, \mathrm{SM}_{\mathrm{DH}}, \alpha_{1}, \alpha_{2}\right)$. The target of calibration is to obtain a vector $\mathbf{V}$, which can minimize the difference between measured and simulated trajectories.

The genetic algorithm is a nonlinear optimization algorithm that simulates biological evolution and has been widely used to solve complex nonlinear optimization problems. It is also used to calibrate the vehicle model [10]. Therefore, we used the genetic algorithm to calibrate the parameter vector $\mathbf{V}$ of the car-following model. With the relative distance, the following vehicle speed and the following vehicle acceleration are common indicators used to evaluate the car-following model, so, we defined the difference parameter $F$ as the fitness function of the genetic algorithm based on these three parameters. The difference parameter $F$ is calculated as follows:

$$
\begin{aligned}
d F(t) & =\frac{\left(\left|v_{s}(t)-v_{r}(t)\right| / v_{r}(t)\right)+\left(\left|d_{s}(t)-d_{r}(t)\right| / d_{r}(t)\right)+\left(\left|a_{s}(t)-a_{r}(t)\right| / a_{r}(t)\right)}{3}, \\
F & =\frac{1}{N} \sum_{t=1}^{N} d F(t)
\end{aligned}
$$

where $v_{s}(t)$ represents the simulated speed of the following vehicle at time $t, v_{r}(t)$ represents the real speed of the following vehicle at time $t, d_{s}(t)$ represents the simulated relative spacing at time $t$, and $d_{r}(t)$ represents the real relative spacing at time $t ; a_{s}(t)$ represents the simulated acceleration of the following vehicle at time $t ; a_{r}(t)$ represents the real acceleration of the following vehicle at time $t ; N$ represents the sampling points number during this carfollowing event. The genetic algorithm is used to find a vector $p$ to minimize the fitness function $F$.

According to the definition of fitness function, one parameter vector $\mathbf{V}$ can be obtained in one car-following event. Therefore, in the extracted 354 car-following events, 354 parameter vectors $\mathbf{V}$ can be obtained, of which unskilled drivers with ADAS OFF have 85 cases, skilled drivers with ADAS OFF have 93 cases, unskilled drivers with ADAS ON have 86 cases, and skilled drivers with ADAS ON have 90 cases. In the next section, these four parameters of the parameter vector $\mathbf{V}$ will carry out $t$-test, respectively.

As shown in Table 3, the test result of $\mathrm{SM}_{\mathrm{DL}}$ shows that there is a statistically significant difference between the $S_{D L}$ of skilled drivers and unskilled drivers during naturalistic driving (ADAS OFF). The $\mathrm{SM}_{\mathrm{DL}}$ of unskilled drivers is significantly affected by the ADAS, and the $\mathrm{SM}_{\mathrm{DL}}$ of skilled drivers is also significantly affected by the ADAS, which indicates that ADAS can significantly affect the drivers' $\mathrm{SM}_{\mathrm{DL}}$. In terms of mean value, the $\mathrm{SM}_{\mathrm{DL}}$ of skilled driver rose from 0.718 to 0.756 after exposure to ADAS, and the $\mathrm{SM}_{\mathrm{DL}}$ of unskilled driver rose from 0.740 to 0.752 after exposure to ADAS, indicating that, in terms of $\mathrm{SM}_{\mathrm{DL}}, \mathrm{ADAS}$ has a greater impact on skilled drivers than unskilled drivers.

As shown in Table 4, the test result of $\mathrm{SM}_{\mathrm{DH}}$ shows that there is a statistically significant difference between the $\mathrm{SM}_{\mathrm{DH}}$ of skilled drivers and unskilled drivers during naturalistic driving. After exposure to ADAS, there is also a statistically significant difference between the $\mathrm{SM}_{\mathrm{DH}}$ of skilled drivers and unskilled drivers. This shows that skilled drivers have higher $\mathrm{SM}_{\mathrm{DH}}$ than unskilled drivers, and ADAS has no significant effect on $\mathrm{SM}_{\mathrm{DH}}$ to both skilled drivers and unskilled drivers.

As shown in Table 5, the test result of $\alpha_{1}$ shows that there is a significant statistical difference between $\alpha_{1}$ of skilled drivers and unskilled drivers during naturalistic driving. After exposure to ADAS, there is also a statistically significant difference between the $\alpha_{1}$ of skilled drivers and unskilled drivers. Unskilled drivers have more rapid acceleration due to insufficient control of the throttle, so the average value of $\alpha_{1}$ is greater than skilled drivers. In terms of $\alpha_{1}$, ADAS has no significant effect on skilled drivers and unskilled drivers.

As shown in Table 6, the test results of $\alpha_{2}$ show that there is a significant statistical difference between $\alpha_{2}$ of skilled drivers and unskilled drivers during naturalistic driving. The $\alpha_{2}$ of unskilled drivers was significantly affected by the ADAS. Unskilled drivers have more sharp decelerations than skilled drivers because of lack of perceived ability, so the $\alpha_{2}$ is greater than that of skilled drivers during naturalistic driving. After exposure to ADAS, the $\alpha_{2}$ of unskilled drivers fall to the same level as skilled drivers'. It is worth 
TABLE 3: T-test result of $\mathrm{SM}_{\mathrm{DL}}$.

\begin{tabular}{|c|c|c|c|c|c|}
\hline & & \multicolumn{2}{|c|}{ ADAS OFF } & \multicolumn{2}{|c|}{ ADAS ON } \\
\hline & & Skilled drivers & Unskilled drivers & Skilled drivers & Unskilled drivers \\
\hline \multirow{2}{*}{ ADAS OFF } & Skilled drivers (mean value: 0.718 ) & - & $0.026^{*}$ & $0.035^{*}$ & - \\
\hline & Unskilled drivers (mean value: 0.740 ) & $0.026^{*}$ & - & - & $0.002^{* *}$ \\
\hline \multirow{2}{*}{ ADAS ON } & Skilled drivers (mean value: 0.756 ) & $0.035^{*}$ & - & - & 0.561 \\
\hline & Unskilled drivers (mean value: 0.752 ) & - & $0.002^{* *}$ & 0.561 & - \\
\hline
\end{tabular}

${ }^{* * *}$ Sig. $<0.001,{ }^{* *}$ sig. $<0.01$, and ${ }^{*}$ sig. $<0.05$.

TABLE 4: T-test result of $\mathrm{SM}_{\mathrm{DH}}$.

\begin{tabular}{lccccc}
\hline & & \multicolumn{2}{c}{ ADAS OFF } & \multicolumn{2}{c}{ ADAS ON } \\
& & Skilled drivers & Unskilled drivers & Skilled drivers & Unskilled drivers \\
\hline \multirow{2}{*}{ ADAS OFF } & Skilled drivers (mean value: 0.718) & - & $\mathbf{0 . 0 1 5}^{*}$ & 0.125 & - \\
& Unskilled drivers (mean value: 0.740$)$ & $\mathbf{0 . 0 1 5}^{*}$ & - & - & 0.224 \\
\hline \multirow{2}{*}{ ADAS ON } & Skilled drivers (mean value: 0.756$)$ & 0.125 & - & $-\mathbf{0 . 0 2 4}^{*}$ & - \\
& Unskilled drivers (mean value: 0.752$)$ & - & 0.224 & $\mathbf{0 . 0 2 4}^{*}$ & - \\
\hline
\end{tabular}

${ }^{* * *}$ Sig. $<0.001,{ }^{* *}$ sig. $<0.01$, and ${ }^{*}$ sig. $<0.05$.

TABLE 5: T-test result of $\alpha_{1}$.

\begin{tabular}{lccccc}
\hline & & \multicolumn{2}{c}{ ADAS OFF } & \multicolumn{2}{c}{ ADAS ON } \\
& & Skilled drivers & Unskilled drivers & Skilled drivers & Unskilled drivers \\
\hline \multirow{2}{*}{ ADAS OFF } & Skilled drivers (mean value: 0.926) & - & $\mathbf{0 . 0 1 7}^{*}$ & 0.235 & - \\
& Unskilled drivers (mean value: 0.974$)$ & $\mathbf{0 . 0 1 7}^{*}$ & - & - & 0.314 \\
\hline \multirow{2}{*}{ ADAS ON } & Skilled drivers (mean value: 0.929) & 0.235 & - & - & $\mathbf{0 . 0 3 3}^{*}$ \\
& Unskilled drivers (mean value: 0.976$)$ & - & 0.314 & $\mathbf{0 . 0 3 3}^{*}$ & - \\
\hline
\end{tabular}

${ }^{* * *}$ Sig. $<0.001,{ }^{* *}$ sig. $<0.01$, and ${ }^{*}$ sig. $<0.05$.

TABLE 6: T-test result of $\alpha_{2}$.

\begin{tabular}{lccccc}
\hline & & \multicolumn{2}{c}{ ADAS OFF } & \multicolumn{2}{c}{ ADAS ON } \\
& & Skilled drivers & Unskilled drivers & Skilled drivers & Unskilled drivers \\
\hline \multirow{2}{*}{ ADAS OFF } & Skilled drivers (mean value: 12.021) & - & $\mathbf{0 . 0 4 4}^{*}$ & 0.235 & - \\
& Unskilled drivers (mean value: 12.884) & $\mathbf{0 . 0 4 4}^{*}$ & - & - & $\mathbf{0 . 0 4 5}^{*}$ \\
\hline \multirow{2}{*}{ ADAS ON } & Skilled drivers (mean value: 12.075) & 0.235 & - & - & 0.033 \\
& Unskilled drivers (mean value: 12.126) & - & $\mathbf{0 . 0 4 5}^{*}$ & 0.033 & - \\
\hline
\end{tabular}

${ }^{* * *}$ Sig. $<0.001,{ }^{* *}$ sig. $<0.01$, and ${ }^{*}$ sig. $<0.05$.

noting that ADAS has no significant effect on skilled drivers in terms of $\alpha_{2}$.

In order to reduce the influence of sampling error on the model, the parameters that have insignificant $T$ test results adopt overall mean. The parameters that have significant $T$ test results adopt mean of each part. The parameter vector is as follows: skilled driver under ADAS OFF $\mathbf{V}_{\mathbf{1}}=(1.3,0.718,0.927$, $6.446,12.072)$, unskilled driver under ADAS OFF $\mathbf{V}_{2}=(1.5$, $0.740,0.975,6.860,12.884)$, skilled driver under ADAS ON $\mathbf{V}_{3}=(0.9,0.754,0.927,6.446,12.072)$, and unskilled driver under ADAS ON $\mathbf{V}_{4}=(1.0,0.754,0.975,6.860,12.072)$.

3.3. Model Validation. In this section, the proposed model is validated in real car-following events data and then compared with the Gazis Herman Rothery (GHR) model. GHR model is an effective car-following model by producing lower percentile errors for speed and acceleration predictions. The GHR model is formulated as follows [36]:

$$
a_{n}(t)=c v_{n}^{m}(t) \frac{v(t-\tau)}{x^{l}(t-\tau)},
$$

where $a_{n}(t)$ is the acceleration of vehicle $n$ (following vehicle) implemented at time $t, V_{n}(t)$ is the speed of the following vehicle, $\Delta x$ and $\Delta v$ refer to the relative distance and speed between the following vehicle and the $(n+1)$ vehicle (leading vehicle), $\tau$ is the driver reaction time, and $m, l$, and $c$ represent the constants.

Four typical types of car-following data $(2(\mathrm{ADAS}) * 2$ (driving experience)) were randomly selected from the database. The simulation results of the proposed model and the GHR model were shown in Figure 2. The Root Mean Square Error (RMSE) was shown in Table 7. From the RMSE 

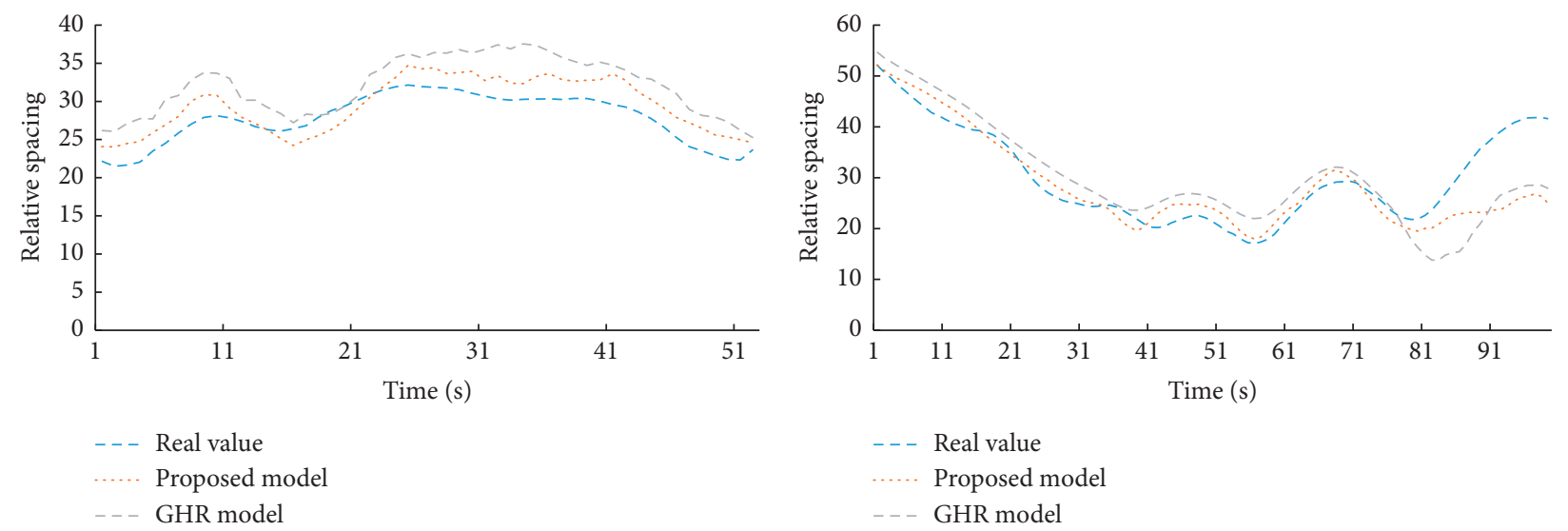

(a)

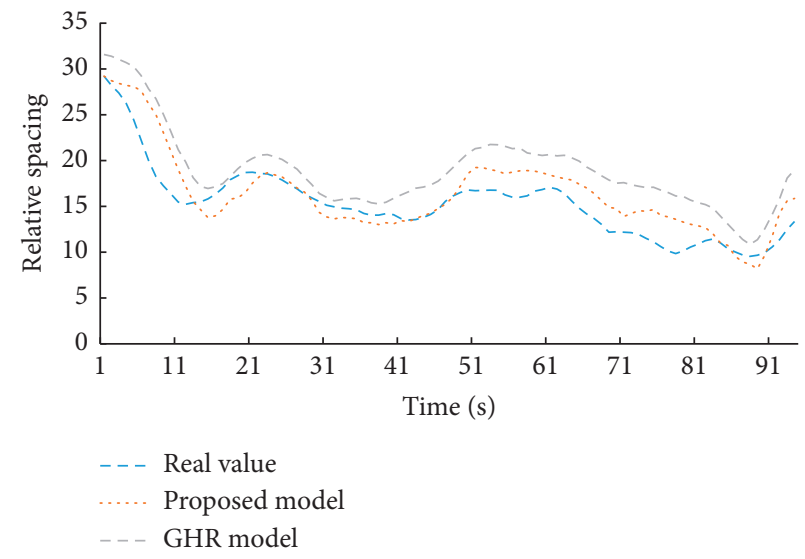

(c)

(b)

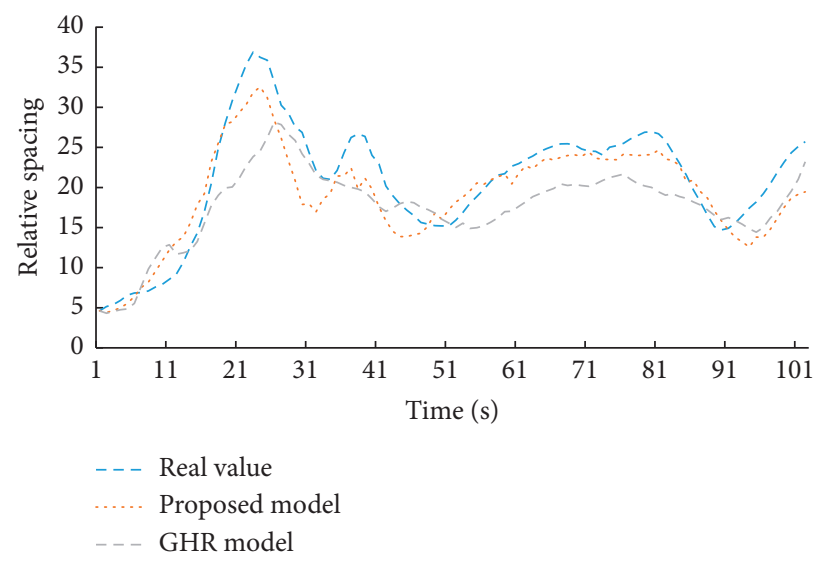

(d)

FIGURE 2: Simulation results of proposed model and GHR model. (a) Skilled driver under ADAS OFF. (b) Unskilled driver under ADAS OFF. (c) Skilled driver under ADAS ON. (d) Unskilled driver under ADAS ON.

TABLE 7: RMSE of the proposed model and the GHR model.

\begin{tabular}{lcccc}
\hline & ADAS OFF & \multicolumn{2}{c}{ ADAS ON } \\
& Unskilled driver & Skilled driver & Unskilled driver & Skilled driver \\
\hline Proposed model & 5.61 & 2.39 & 3.26 & 2.29 \\
GHR mode & 6.68 & 4.68 & 4.72 & 4.08 \\
\hline
\end{tabular}

of the simulation results, it can be seen that the proposed model is better than the GHR model.

\section{Conclusion}

In this study, a total of 354 car-following events were extracted from 87 times of FOTs. Based on these data, we established a car-following model using SM as the risk level quantization parameter based on risk homeostasis theory. Firstly, three-way ANOVA was used to analyze the three independent variables (ADAS, driving experience, and gender). The results showed that ADAS and driving experience have a significant effect on the drivers' car-following behavior. Then, according to these two significant factors, the car-following model was established. In the calibration process of the five model parameters $\left(\tau, \mathrm{SM}_{\mathrm{DL}}, \mathrm{SM}_{\mathrm{DH}}, \alpha_{1}\right.$, and $\alpha_{2}$ ), the statistical method was used to calibrate the first parameter reaction response $\tau$. The remaining four parameters were calibrated using a classical genetic algorithm, and the effects of ADAS and driving experience on these four parameters were analyzed using $T$-test. Finally, the proposed model was compared with the GHR model, which was adopted by most ACC systems. The result showed that the proposed model has smaller RMSE than the GHR model. The proposed model is a method for simulating different driving behaviors that are affected by ADAS and individual 
characteristics. Considering more driver individual characteristics, such as driving style, is the future researches goal.

\section{Data Availability}

The data used to support the findings of this study are available from the corresponding author upon request.

\section{Conflicts of Interest}

The authors declare no conflicts of interest.

\section{Acknowledgments}

This research was funded by the National Nature Science Foundation of China (Nos. 51775396 andNo. 52072290), Hubei Province Science Fund for Distinguished Young Scholars (No. 2020CFA081), and the Fundamental Research Funds for the Central Universities (No. 191044003, No. 2020-YB-028).

\section{References}

[1] World Health Organization, Global Status Report on Road Safety 2017, World Health Organization, Geneva, Switzerland, 2017.

[2] J. Mar and H.-T. Lin, "A car-following collision prevention control device based on the cascaded fuzzy inference system," Fuzzy Sets and Systems, vol. 150, no. 3, pp. 457-473, 2005.

[3] J. Wang, L. Zhang, D. Zhang, and K. Li, "An adaptive longitudinal driving assistance system based on driver characteristics," IEEE Transactions on Intelligent Transportation Systems, vol. 14, no. 1, pp. 1-12, 2013.

[4] S. Panwai and H. Dia, "Comparative evaluation of microscopic car-following behavior," IEEE Transactions on Intelligent Transportation Systems, vol. 6, no. 3, pp. 314-325, 2005.

[5] C. Wang and B. Coifman, "The effect of lane-change maneuvers on a simplified car-following theory," IEEE Transactions on Intelligent Transportation Systems, vol. 9, no. 3, pp. 523-535, 2008.

[6] E. Kometani and T. Sasaki, "Dynamic behavior of traffic with a nonlinear spacing-speed relationship," in Proceedings of the Symposium on Theory of Traffic Flow, Research Laboratories, General Motors, pp. 105-119, New York, NY, USA, 1959.

[7] R. E. Chandler, R. Herman, and E. W. Montroll, "Traffic dynamics: studies in car following," Operations Research, vol. 6, no. 2, pp. 165-184, 1958.

[8] S. H. Hamdar, M. Treiber, H. S. Mahmassani, and A. Kesting, "Modeling driver behavior as sequential risk-taking task," Transportation Research Record: Journal of the Transportation Research Board, vol. 2088, no. 1, pp. 208-217, 2008.

[9] G. J. S. Wilde, "The theory of risk homeostasis: implications for safety and Health," Risk Analysis, vol. 2, no. 4, pp. 209-225, 1982.

[10] G. Lu, B. Cheng, Y. Wang, and Q. Lin, “A car-following model based on quantified homeostatic risk perception," Mathematical Problems in Engineering, vol. 2013, Article ID 408756, 13 pages, 2013.

[11] Y. Saito, M. Itoh, and T. Inagaki, "Driver assistance system with a dual control scheme: effectiveness of identifying driver drowsiness and preventing lane departure accidents," IEEE Transactions on Human-Machine Systems, vol. 46, no. 5, pp. 1-12, 2016.
[12] J. Son, M. Park, and B. B. Park, "The effect of age, gender and roadway environment on the acceptance and effectiveness of advanced driver assistance systems," Transportation Research Part F: Traffic Psychology and Behaviour, vol. 31, pp. 12-24, 2015.

[13] C. Blaschke, F. Breyer, B. Färber, J. Freyer, and R. Limbacher, "Driver distraction based lane-keeping assistance," Transportation Research Part F: Traffic Psychology and Behaviour, vol. 12, no. 4, pp. 288-299, 2009.

[14] S. A. Birrell, M. Fowkes, and P. A. Jennings, "Effect of using an in-vehicle smart driving aid on real-world driver performance," IEEE Transactions on Intelligent Transportation Systems, vol. 15, no. 4, pp. 1801-1810, 2014.

[15] E. Adell, A. Várhelyi, and M. D. Fontana, "The effects of a driver assistance system for safe speed and safe distance-a real-life field study," Transportation Research Part C: Emerging Technologies, vol. 19, no. 1, pp. 145-155, 2011.

[16] N. Lyu, Z. Duan, L. Xie, and C. Wu, "Driving experience on the effectiveness of advanced driving assistant systems," in Proceedings of the 2017 4th International Conference on Transportation Information and Safety (ICTIS), pp. 987-992, Banff, Canada, August 2017.

[17] N. Lyu, Z. Duan, C. Ma, and C. Wu, "Safety margins - a novel approach from risk homeostasis theory for evaluating the impact of advanced driver assistance systems on driving behavior in near-crash events," Journal of Intelligent Transportation Systems, vol. 25, no. 1, pp. 93-106, 2021.

[18] N. Lyu, C. Deng, L. Xie, C. Wu, and Z. Duan, "A field operational test in China: exploring the effect of an advanced driver assistance system on driving performance and braking behavior," Transportation Research Part F: Traffic Psychology and Behavior, vol. 65. , 2018 In press.

[19] N. Lyu, Z. Duan, and C. Wu, "The impact of driving experience on advanced driving assistant systems," Journal of Transportation Systems Engineering and Information Technology, vol. 17, no. 6, pp. 48-55, 2017.

[20] I. Engströme, N. P. Gregersen, K. Hernetkoski, E. Keskinen, and A. Nyberg, Jeunes Conducteurs Novices, Education \& Formation du Conducteur. Etude bibliographique. Rapport VTI 491A, Université de Turku, Turku, Finland, 2003.

[21] G. Underwood, P. Chapman, N. Brocklehurst, J. Underwood, and D. Crundall, "Visual attention while driving: sequences of eye fixations made by experienced and novice drivers," Ergonomics, vol. 46, no. 6, pp. 629-646, 2003.

[22] L. L. D. Stasi, V. Álvarez-Valbuena, J. J. Cañas, A. Maldonado, A. Catena, and A. Antolí, "Risk behavior and mental workload: multimodal assessment techniques applied to motorbike riding simulation," Transportation Research Part F: Traffic Psychology and Behavior, vol. 12, no. 5, pp. 361-370, 2009.

[23] C. Maag, D. Muhlbacher, and H.-P. Mark, "Studying effects of advanced driver assistance systems (ADAS) on individual and group level using multi-driver simulation," IEEE Intelligent Transportation Systems Magazine, vol. 4, no. 3, pp. 45-54, 2012.

[24] D. J. Leblanc, S. Bao, J. R. Sayer, and S. Bogard, "Longitudinal driving behavior with integrated crash-warning system," Transportation Research Record: Journal of the Transportation Research Board, vol. 2365, no. 1, pp. 17-21, 2013.

[25] L. Chong, M. M. Abbas, A. Medina Flintsch, and B. Higgs, "A rule-based neural network approach to model driver naturalistic behavior in traffic," Transportation Research Part C: Emerging Technologies, vol. 32, no. 4, pp. 207-223, 2013.

[26] R. Ervin, J. Sayer, D. Leblanc, S. Bogard, and M. Mefford, Automotive Collision Avoidance System Field Operational Test 
Report, University of Michigan Ann Arbor Transportation Research Institute, Ann Arbor, MI, USA, 2005.

[27] X. Wang, M. Zhu, and Y. Xing, "Impacts of collision warning system on car following behavior based on naturalistic driving data," Journal of Tongji University (Natural Science), vol. 44, no. 7, pp. 1045-1051, 2016.

[28] G. Lu, B. Cheng, Q. Lin, and Y. Wang, "Quantitative indicator of homeostatic risk perception in car following," Safety Science, vol. 50, no. 9, pp. 1898-1905, 2012.

[29] G. J. S. Wilde, "Critical issues in risk homeostasis theory," Risk Analysis, vol. 2, no. 4, pp. 249-258, 2010.

[30] R. Nilsson, Safety Margins in the Driver. The Faculty of Social Sciences, Uppsala University, Uppsala, Sweden, 2001.

[31] X. Zhang, G. Lu, and B. Cheng, "Parameters calibration for car-following model based desired safety margin," International Conference on Optoelectronics and Image Processing (ICOIP), vol. 2, pp. 97-100, 2010.

[32] T. L. Brown, J. D. Lee, and D. V. Mcgehee, "Human performance models and rear-end collision avoidance algorithms," Human Factors: The Journal of the Human Factors and Ergonomics Society, vol. 43, no. 3, pp. 462-482, 2001.

[33] G. S. Gurusinghe, T. Nakatsuji, Y. Azuta, P. Ranjitkar, and Y. Tanaboriboon, "Multiple car-following data with real-time kinematic global positioning system," Transportation Research Record: Journal of the Transportation Research Board, vol. 1802, no. 1, pp. 166-180, 2002.

[34] H. Ozaki, "Reaction and anticipation in the car-following behavior," Transportation and Traffic Theory, vol. 49, no. 6, pp. 2302-2306, 1993.

[35] N. Lyu, Z. Duan, and C. Wu, Evaluated the Effectiveness of Advanced Driving Assistant Systems in Near-Crash Events Based on Safety Margin, in Proceedings of the 97st Transportation Research Board Annual Meeting, Washington, DC, USA, 2018.

[36] M. Brackstone and M. McDonald, "Car-following: a historical review," Transportation Research Part F: Traffic Psychology and Behaviour, vol. 2, no. 4, pp. 181-196, 1999. 